\title{
Forensic Method to Verify the Genuineness of a Disputed Signature
}

\author{
Amitabh Verma
}

\begin{abstract}
The genuineness of the document can be proved by any witness who believe it to be the handwriting of such person because he saw the person executed it. When comparing a forged signature with a series of actual signature of the same person whose signature is being questioned, the general presentation and pictorial impact of the writing will propose, as the proportion of likenesses or contrasts prevails, an impression upon the mind of the analyst with regards to the certified or forged character of the signature in question. When it is comprehended that to make an imitation accessible for the purposes behind its creation it must resemble the composition of the individual whose signature it indicates to represent, it follows as a reasonable conclusion that the similarities all in all appearances alone should be secondary factors in setting up the validity of a signature by correlation and the way that two signature resemble the other alike isn't generally prove that they were composed by a similar individual. The area of handwritten signature genuineness has been broadly researched in the last decades. In this paper I present how the genuineness of a signature can be proved.
\end{abstract}

Index Terms-Signatures, Forgery, Genuineness, questioned document, Disputed, Imitation

\section{INTRODUCTION}

Signature has been a distinctive element for individual identification through ages. Signature are a special instance of hand writing in which unique characters and twists are accessible. By and large, the signature isn't meaningful even by a human. It is a behavioural measurement of life. It did not depend on physiological properties of the individual, for example, fingerprint or face, yet behavioural. In that capacity one's signature may change after some time and it isn't so special or difficult to forge as iris examples or fingerprints. Nonetheless, signature acceptance by the public makes it suitable for certain lower-security validation needs. Signature examination must be connected when the individual is/was conscious and disposed to write in the typical manner. In this paper a forensic approach method for the proof of signature genuineness present.

Manuscript revised on January 15, 2019 and published on January 26, 2019

Amitabh Verma, Department of Management, Birla Institute of Technology, Mesra, Ranchi, Jharkhand, India

\section{RELATED WORK}

A. Herkt,1986 described and compared the disguise habits and methods of forgery, and the most common failings in the quality of the forgery attempts.

Katrin Franke,2009 described empirical studies of kinematic and kinetic signature characteristics. It is a more in-depth analysis which reveals insides on differences and similarities of authentic and mimicked signing movements and also discussed implications for ink-trace characteristics on paper. Carolyne Bird, Bryan Found , Kaye N Ballantyne,2009 examined the opinion of forensic handwriting examiners regarding the 'process of production' (which in this case was a choice between written naturally or written using a disguise/simulation strategy) of the questioned disguised and simulated signatures in blinded skill testing trials and the results showed the usefulness of FHEs (forensic handwriting examiners offering a first stage simulation/disguise process opinion without going on to form an opinion on authorship, as the support for the proposition that a signature is something other than genuine may be, in itself, of strong evidential value.

Bryan Found, Doug Rogers,2008 investigated the character of Forensic Handwriting Examiners' (FHEs) authorship opinions on questioned signatures through the medium of blind validation trials. Results showed that, as a group, FHEs were significantly more confident at identifying writers' genuine signatures than identifying writers' disguised signatures or eliminating specimen writers from having authored simulated signatures.

There are few numbers of papers on the genuineness of a signatures. Generally, the papers are related to the proposed method on signature identifications process.

\section{GENUINENESS OF A Signature}

In examining alleged forgery, it is important to remember that proof of validity of all types is in all ways just the inverse of proof of evidence. The most common types of effects of fraud are not as is incorrectly thought by numerous divergences in structure however a drawn and hesitating nature of stroke or time especially at specific parts of letters that should be freely written, showing that during the writing demonstration consideration was given to the details of writing and normally the opposite condition are those that show genuineness.

Flying begins and flying completions were the movement of the pen demonstrates the start of the stroke and continues beyond the end to vanishing point are found in free normal writing, and as a rule, are vital signs of validity. Intermediate strokes also where the pen comes off the paper yet isn't 
stopped and demonstrates continuity of movement are as a rule, signs of unconsciousness of the details of the activity and point toward validity. Anything of any kind about a writing that indicate that the writer was not thinking about the writing itself is evidence of genuineness.

When writing shows by any quality or in any way that it is the result of unconscious habit this always is a forceful indication of genuineness. This quality is shown by repeated significant characteristics executed with ordinary attention to the operation as indicated by incompleteness, illegibility natural variation and the carelessness.

Shortened, mutilated and obscured shapes, which are adequately free and fast regularly really show genuineness. The individuals who compose with trouble or hesitation through some physical sickness may sometimes delivers broken and incomplete signature and these outcomes which in themselves are distinctly unique as compared with signatures created under state of strength and health, may forcefully demonstrate validity.

Under states of weakness because of illness or age, portions of a real signature might be awkwardly composed over a second time not at just the similar spot and in a way which clearly demonstrates that the writer either couldn't see or was to weak and inattentive as not to cares what the outcome may be. This careless perfectly apparent redundancy of the frauds frequently show validity.

Unusual illegibility should generally be constructed as evidence of genuineness. The failure to rewrite or correct obvious ink failures in an illegible or incomplete signature is another indication of genuineness.

A conclusion of genuineness result when the writing under investigations contains a sufficient combination of naturally written qualities and characteristics so that it is unreasonable to say that they would all be present in a forgery.

When a suspected and a standard writing are alike in a delicate line quality which is due to habitual delicate variations in pen pressure or what has here to far been described as unconscious shading, this conformity often is strong evidence of genuineness, This quality of line or the characteristics of the strokes themselves is an important but somewhat elusive quality in writing and very difficult to imitate successfully.

If standard genuine signatures are skilfully shaded and an alleged imitation of the writing contains correct well-placed shadings, especially shadings on curved strokes in which there is a gradual release of pressure from a heavy to a time line made with a rapidly moving pen. This condition in writing also is evidence of genuineness. This particular element of writing skilful shading, requires correct pen position and a high degree of manual skill and it points to a largely unconscious writing habit not consistent with the theory of copying, drawing, tracing or simulation. If throughout a questioned document of considerable length all these variations in width of stroke conform to the standard writing of the one whose writing is under examination these qualities are strong evidence of genuineness.

Another sign of validity is a holographic record or a significantly amount of writing, or in at least two signatures are certain regular varieties in the details of the writing. It is difficult for the unpractised examiner to understand that a specific degree of varieties in a group of a few signatures and varieties in repeated words and letters in a continues holographic document can be proof of validity.

\section{STANDARDS OF COMPARISON}

One of the first step in the investigation of a suspected or disputed writing should be the seeking out of a suitable genuine writing with which it is to be compared.

The best standard of examination is those of indistinguishable general class from the questioned document and as nearly as possible of a similar date. The measure of writing necessary for comparison in various cases however if possible, enough should always be acquired to demonstrate clearly the writing habits for the one whose writing is under scrutiny.

A positive decision that a signature is deceitful can sometimes be reached by examination with a little amount of genuine writing exceptionally as expressed above, if the questioned signature is a mishandling imitation that is suspicious. Increasingly standard writing may therefore be essential as a reason for a positive assessment that a writing is real than is important to demonstrate that it is fraudulent.

A few signatures should always be acquired if possible before any ultimate conclusion is rendered, five signatures always comprising a more satisfactorily reason for an opinion than one and ten being superior to five. It is not often helpful to use more than twenty. Five to seventy-five exception of in unusual cases and it isn't typically desirable to utilize these of broadly different dates if adequate contemporary writings of the correct class can be obtained. In many cases a few contemporary signatures furnish an adequate basis for a positive opinion and with certain signatures and skilful writers are good standard signatures is sometimes enough on which to base a preliminary opinion.

It is dangerous to base a positive and final decision that a questioned signature is certified on an examination of it with just a single authentic signature except if it is an exceedingly individualized and skilful signature.

A suspected signature however may contain so many inherent qualities indicating that it is not genuine that is one good standard signature may be sufficient on which to base a positive opinion that it is not genuine.

As expressed over the writings most to be relied on as standard are always those bearing dates closest to the date of the questioned writing and that are of a similar general class. This is valid for the reason that writing of various persons fluctuates in varying degrees as written at various occasions and for various purposes and the best way to learn of these habits for any writer through standard writing.

A very nervous and physically weak writer may become wholly unable to write under conditions of excitement or strain, but the inability is not due merely to the importance of the document. Furthermore, nervous writers do not produce the qualities found in the typical forgery. Some of the standard questions of comparison for signature verification/genuineness are

- Is the signature genuine?

- Is the signature in a natural position?

- Is the witness signature genuine and were they written in the order they appear? 
- Does the signature touch any of the writing and was the signature written last?

- Is the writing of the body of the document genuine?

- Is any of the writing disguised or unnatural in any way?

- Are the standards submitting all genuine and of proper date?

- Are the remains of pencil or carbon marks which may have been an outline for the signature or other writing?

- Is the signature shown in embossed form on the back of the sheet?

- Is the document written before the paper was folded?

- Is the signature written before or after the paper was folded?

- How many times and in what way was the document folded?

- Is there any significance in the design of the signature as indicating its date?

- Was there one kind of ink used in the preparation of the document?

- Is the apparent age of the writing ink used consistent with the date of the document?

- What kind of paper was used and does its size, shape, colour or constituent have any date or origin significance?

- Are the several sheets of the document exactly the same size, thickness and colour?

- Does the paper ruling have any significance?

- Was the paper torn, burned or mutilated in any way and it so far what purpose?

- Is the paper of an unusual size and is it intended on any margin and was it trimmed or cut by hand at any place?

- Was the paper stapled or otherwise fastened together more than once?

- Is the paper unnecessarily crumpled?

- Are these discolouration or stains on the paper and if so what probably caused them and are they natural or artificial?

- Does the document contain chemical or pencil erasures, alteration or substitution of any kind?

- Does the document show lack of continuity when viewed by transmitted light?

- Are these effects of ink writing from other sheets of paper?

- Was the document copied in a wet copying press?

- Has the document been wet and if so, for what purpose?

- Have accidental pencil or other marks been made on the paper?

- If typewritten was it all written on the same machine?

- What kind of machine was used?

- Was each sheet written continuously without being removed from the typewriter?

- Is the history of the machine consistent with the date of the document?
- Are these added figure, words, clauses, sentences, paragraphs or pages written on a different typewriter?

- Is the typewriting the work of a skilled operator?

- Are the margins, punctuations and spacing uniform?

- Was the typewriting written by the operator alleged to have written it?

- Is the connection of the pages continuous in language and ribbon condition consistent with other work on the machine on the same date?

- If document is a carbon copy does it conform in size, position and arrangement of matter with original letter heads?

- Does the printing or ruling on the document have any date or other significance?

- If document is a letter does postmark, postage stamp cancellation stamp or embossing on enclosure have any significance?

The goal of handwriting analysis is to answer these questions about a suspicious document and determine authorship using a variety of scientific methods. Methods are based on the principle of identification. Handwriting expert compare handwriting characteristics of a questionable document to those of a known sample to try to determine if the same person wrote the document. This analysis also helps to detect forgeries.

\section{Conclusion}

Although it is still important that handwriting evidence be used in combination with other sources of evidence, handwriting analysis is considered a reproducible and peer-reviewed scientific process. This paper has displayed a precise forensic examination technique for genuineness. The limitation are: Although an experienced questioned document examiner can detect many cases of forgery, some may be missed. The quality of standards obtained often determines the quality of a comparison analysis and good standards may be difficult to obtain. Another limitation is the effects of mood, age, drugs, fatigue and illness on a person's handwriting/signatures.

\section{CONFLICT OF INTEREST}

The author whose name is listed certify that they have NO affiliations with or involvement in any organization or entity with any financial interest (such as honoraria; educational grants; participation in speakers' bureaus; membership, employment, consultancies, stock ownership, or other equity interest; and expert testimony or patent-licensing arrangements), or non-financial interest (such as personal or professional relationships, affiliations, knowledge or beliefs) in the subject matter or materials discussed in this manuscript..

\section{REFERENCES}

[1] A.Piyush Shanker, A. N. Rajagopalan, Off-line signature verification using DTW , In: Pattern Recognition Letters, Vol. 80, 2016, pages $84-90$

[2] Amir Soleimani, Babak N. Araabi , Kazim Fouladi, Deep Multitask Metric Learning for offline Signature Verification , In: Pattern Recognition Letters, vol. 80, 2016, pages 84-90 


\section{Available online at www.ijrat.org}

[3] Ali Karouni, Bassam Daya, Samia Bahlak, Offline signature recognition using neural networks approach, In: Procedia Computer Science, vol. 3, 2011,pages 155-161.

[4] Pradeep N. Narwade, Rajendra R. Sawant, Sanjiv V. Bonde, Offline signature verification using shape correspondence, In: International Journal of Biometrics, vol. 10, 2018, pages 272-289

[5] Anh Pham, Hong-Ha Le, Nang-Toan, Offline handwritten signature verification using local and global features, In: Annals of Mathematics and Artificial Intelligence, vol 75,2015, pages 231-247.

[6] Anil K. Jain, Friederike D. Griess ,Scott D. Connell, On-line signature verification, In: Pattern Recognition, vol. 35 ,2002. pages 2963 - 2972

[7] Carolyne Bird, Bryan Found, Kaye N Ballantyne, Doug Rogers, Forensic handwriting examiners' opinions on the process of production of disguised and simulated signatures, In: Forensic science international, 2010, pages 103-107.

[8] Jacques Mathyer, The Expert Examination of Signatures, In: Journal of Criminal Law and Criminology 1961, volume 52, pages 122-126.

[9] GA Dawson, BS Lindblom, An evaluation of line quality in photocopied signatures Centre of Forensic Sciences, In: Science \& Justice, 1998, volume 38, pages 189-194.

\section{AUTHORS PROFILE}

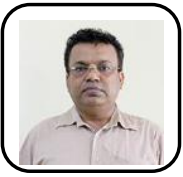

Amitabh Verma is working as Assistant Professor, Department of Management, BIT, Mesra, India. He received his Master of Computer Application, Ph.D. in Management in 2015. He has over 13 years of experience in both teaching and research. His research interest includes DSS, Database Management, Data Mining, MIS, E-Business, AI \& Cloud Computing. He has published research papers in international journals and conferences. He is a member of IAENG (International Association of Engineers) and a life member of Indian Society for Technical Education.
[10] A. Herkt, Signature Disguise or Signature Forgery? In: Journal of the Forensic Science Society, 1986, volume 26, pages 257-266.

[11] Katrin Franke, Analysis of Authentic Signatures and Forgeries, In: International Workshop on Computational Forensics IWCF, 2009, pages 150-164.

[12] Bryan Found, Doug Rogers, The probative character of Forensic Handwriting Examiners' identification and elimination opinions on questioned signatures In: Forensic Science International, 2008, volume 178, pages 54-60. 